UWThPh-1998-40

PRL-TH-1998

September 1998

\title{
The field-theoretical approach to coherence in neutrino oscillations
}

\author{
W. Grimus and P. Stockinger \\ Institute for Theoretical Physics, University of Vienna \\ Boltzmanngasse 5, A-1090, Vienna, Austria \\ S. Mohanty \\ Theory Group, Physical Research Laboratory \\ Ahmedabad - 380 009, India
}

\begin{abstract}
We study the conditions for the existence of neutrino oscillations in the field-theoretical approach which combines neutrino production and detection processes in a single Feynman graph. The "oscillating neutrino" is represented by an inner line of this graph where, due to the macroscopic distance $L$ between source and detector, the neutrino propagators for neutrinos with definite mass are replaced by the projection operators unto the neutrino states on mass shell. We use as a concrete model reaction the neutrino source and detector as given in the LSND experiment and we carefully take into account the finite lifetime of the stopped muons which provide the $\bar{\nu}_{\mu}$ beam. We show that the field-theoretical approach provides a solid method to locate all possible conditions and allows to separate unambiguously their different origins. Some of these conditions are independent of $L$ whereas others state that coherence is lost when $L$ exceeds a certain "coherence length". Also it turns out that, at least in the concrete situation considered here, the concept of neutrino wave packets is not supported by the field-theoretical approach for realistic experimental conditions, i.e., the neutrino energy spread is incoherent in origin.
\end{abstract}

14.60.Pq, 03.65.-w

Typeset using REVTEX 


\section{INTRODUCTION}

The standard treatment of neutrino oscillations [1],2] provides a beautiful and simple picture of this important phenomenon. With the mixing matrix relating the left-handed neutrino flavor fields with the left-handed neutrino mass eigenfields defined by $\nu_{L \alpha}=\sum_{j} U_{\alpha j} \nu_{L j}$ $(\alpha=e, \mu, \tau, \ldots)$ it allows to derive the oscillation probabilities for antineutrinos

$$
\begin{aligned}
P_{\bar{\nu}_{\alpha} \rightarrow \bar{\nu}_{\beta}} & =\left|\sum_{j} U_{\beta j}^{*} U_{\alpha j} \exp \left(-i \frac{m_{j}^{2} L}{2 E_{\nu}}\right)\right|^{2} \\
& =\sum_{j}\left|U_{\beta j}\right|^{2}\left|U_{\alpha j}\right|^{2}+2 \operatorname{Re}\left\{\sum_{j>k} U_{\beta j}^{*} U_{\alpha j} U_{\beta k} U_{\alpha k}^{*} \exp \left(-i \frac{\Delta m_{j k}^{2} L}{2 E_{\nu}}\right)\right\},
\end{aligned}
$$

valid in the ultrarelativistic limit with $\Delta m_{j k}^{2} \equiv m_{j}^{2}-m_{k}^{2}$ where $m_{1} \leq m_{2} \leq \ldots$ denote the neutrino masses, $L$ is the distance between neutrino source and detector and $E_{\nu}$ is the neutrino energy. The probability for neutrinos is obtained from Eq.(1.1) by the substitution $U \rightarrow U^{*}$. In the following, Greek indices always indicate neutrino flavors and Latin indices mass eigenstates or fields. However, after a closer look one discovers that the standard derivation of Eq.(1.1) needs clarification in several points (see, e.g., Ref. [3] for a summary of these problems). This has first been attempted by using neutrino wave packets [1: 12, whereas Ref. [3] has pioneered the idea of considering the complete neutrino production detection chain using only those quantities for the description of neutrino oscillations which are really observed or manipulated in oscillation experiments 13 15 in order to obtain unambiguous results. See also Ref. [16] for a sort of combined field theory - wave packet approach. The present interest in theoretical treatments of neutrino oscillations can be phrased by the following question: Under which conditions is formula (1.1) valid? Since the neutrino wave packet formalism does not work with the physical observables we find the fieldtheoretical approach treating neutrino production and detection [13] the most appropriate, unambiguous and general way to analyse the problem of coherence in neutrino oscillations. In particular, when several quantities defining a length are involved, an improvement of the wave packet approach is called for in order to distinguish the roles and origins of these lengths.

The transition probability (1.1) is given by the square of the sum over the amplitudes of the neutrino mass eigenstates, i.e., by a coherent summation over the mass eigenstates. The first term in the second line of Eq.(1.1) represents the purely incoherent summation over the mass eigenstates whereas the second term denotes the interference terms. The exponentials $\exp \left(-i 2 \pi L / L_{j k}^{\mathrm{osc}}\right)$ with the oscillation lengths defined by

$$
L_{j k}^{\mathrm{osc}} \equiv \frac{4 \pi E_{\nu}}{\Delta m_{j k}^{2}}
$$

show the oscillatory behaviour of the transition probability as a function of $L / E_{\nu}$. Eq.(1.1) is a theoretical expression without regard to an actual experimental situation. In the description of a neutrino oscillation experiment it is possible that, after taking into account the experimental conditions, some or all of the interference terms drop out as a consequence 
of certain averaging or suppression mechanisms to be discussed in the following. Note that the effect of such mechanisms is equivalent to a partial or complete incoherent summation over the neutrino mass eigenstates.

One such class of mechanisms is given by all effects leading to an energy spread of the neutrino beam. It has been shown in Ref. [7 that if we label such effects by $a$ then each of these effects giving an energy spread $\Delta E_{a}$ leads to a coherence length

$$
L_{a ; j k}^{\mathrm{coh}}=L_{j k}^{\mathrm{osc}} \frac{E_{\nu}}{\Delta E_{a}}
$$

independent of the fact whether this spread has to be interpreted as a coherent or incoherent effect. In the context of a neutrino energy spread "incoherent" means that single neutrinos have a definite energy but the neutrino beam has an energy spread whereas by "coherent" it is understood that a single neutrino state is a superposition of different energies." Note, however, that in the field-theoretical approach the notions "incoherent" and "coherent" energy spread have well-established and precise definitions: "incoherent" means that the summation over different neutrino energies happens in the cross section of the total production - detection process whereas a summation over different neutrino energies in the amplitude is called a "coherent" energy spread. 'f In the following we adhere to these definitions and refer the reader to section $\nabla]$ for a clarification of the notion of neutrino energy in the field-theoretical approach where the oscillating neutrinos occur in an inner line of the combined production - detection Feynman graph. Having different oscillation lengths in the process under discussion, then clearly the relevant coherence length is given by [17]

$$
L_{j k}^{\mathrm{coh}} \equiv \min _{a} L_{a ; j k}^{\mathrm{coh}} .
$$

Both kinds of neutrino energy spread, coherent and incoherent, lead to a loss of the oscillation pattern if $L>L_{j k}^{\mathrm{coh}}$ and cannot be distinguished experimentally [7]. Apart from the condition $L \lesssim L_{j k}^{\text {coh }}$ other conditions have to be fulfilled for the oscillation pattern to be present which do not depend on $L$ [3, 13, 14].

In this paper we will use the LSND experiment [18 with the process

$$
\mu^{+} \rightarrow e^{+}+\nu_{e}+\bar{\nu}_{\mu} \stackrel{\nu \text { osc. }}{\rightarrow} \bar{\nu}_{e}+p \rightarrow n+e^{+}
$$

as a model for our investigation for two reasons: first of all, the $\bar{\nu}_{\mu}$ neutrino source $\left(\mu^{+}\right)$is unstable and we want to extend the field-theoretical approach of Ref. [13] by taking into account the finite lifetime of the source; secondly, there is a claim made in Ref. [19] that in the LSND experiment the condition for coherence is not fulfilled. In the following, we will discuss in detail the effects of

\footnotetext{
${ }^{1}$ The notion of a neutrino wave packet is synonymous with the presence of a coherent neutrino energy spread.

${ }^{2}$ Hence, whether the summation over neutrino mass eigenstates or the summation over neutrino energies is concerned, "coherent" refers to a summation in the amplitude whereas "incoherent" refers to a summation over squares of amplitudes.
} 
1. the quantum-mechanical uncertainties of momentum and energy of the initial particles involved in the production and detection processes represented by the widths of their respective wave packets or stationary states,

2. the finite lifetime of the neutrino source particle,

3. the uncertainties in the measurements of energies and momenta of the particles in the final state of neutrino production and, in particular, of the detection process.

We are not able to take into account the interaction of the neutrino source particle (the $\mu^{+}$ in our case) or the interaction of particles in the final state of the source process (in our case the positron originating from the $\mu^{+}$decay) with the matter background in which the source particle is generated (in LSND this background is water) in the field-theoretical treatment. We will only comment on the second of these effects in the last section of the paper. In the wave packet approach it is said that the interaction of the particles in the source process with the matter background interrupt neutrino emission and estimates of these effects are used to determine the "size of the neutrino wave packet" [2].

To include the finite lifetime of the neutrino source of the process (1.5) we combine field theory with the Weisskopf-Wigner approximation [20] in section [1]. In section ПI] we calculate the amplitude for the reaction (1.5) by taking into account that the distance $L$ between the source and the detector is macroscopic. This is achieved by using a theorem proved in Ref. [13 and an integral discussed in detail in the appendix of the present paper. In section $[\mathrm{IV}$ we derive conditions for the existence of neutrino oscillations independent of $L$, while in section $\nabla$ we study some aspect of the cross section of the total production detection process concerning the finite lifetime of the source. All the conditions for neutrino oscillations obtained in sections $\square$ and $\nabla$ - whether dependent on $L$ or not - are discussed in detail in section V1 where we also study the problem raised in Ref. [19] and try to elucidate the nature of the neutrino energy spread.

\section{PERTURBATION THEORY AND WEISSKOPF-WIGNER APPROXIMATION}

To fix the notation we shortly repeat the basics of time-dependent perturbation theory. We consider a system described by the Hamiltonian $H=H_{0}+H_{1}$ where $H_{0}$ and $H_{1}$ are not not explicitly time-dependent. The eigenvalues and eigenvectors of $H_{0}$ will be denoted as in the relation $H_{0} \phi_{j}=E_{j} \phi_{j}$ where $\left\{\phi_{j}\right\}$ is a complete orthonormal system of states. For an arbitrary state $\psi=\sum_{j} c_{j}(0) \phi_{j}$ at $t=0$, the Schrödinger Equation gives the time evolution $\psi(t)=\sum_{j} c_{j}(t) \phi_{j} e^{-i E_{j} t}$ where the components $c_{j}(t)$ obey the relations

$$
i \dot{c}_{j}(t)=\sum_{k} c_{k}\left\langle\phi_{j} \mid H_{1} \phi_{k}\right\rangle e^{i\left(E_{j}-E_{k}\right) t}=\sum_{k} c_{k}\left\langle\phi_{j} \mid H_{1, \mathrm{int}}(t) \phi_{k}\right\rangle
$$

and

$$
H_{1, \text { int }}(t) \equiv e^{i H_{0} t} H_{1}(0) e^{-i H_{0} t}
$$


defines the interaction Hamiltonian in the interaction picture.

Let us now study the $\mu^{+}$decay and the "subsequent" detection of $\bar{\nu}_{e}$ by $\bar{\nu}_{e}+p \rightarrow e^{+}+n$. The interaction Hamiltonian is given by

$$
H_{1}=H_{S}^{+}+H_{S}^{-}+H_{D}^{+}+H_{D}^{-}
$$

where the indices $S$ and $D$ denote source and detection, respectively, and $H_{S}^{+}$and $H_{D}^{+}$are given by the Hamiltonian densities

$$
\begin{aligned}
\mathcal{H}_{S}^{+} & =\frac{G_{F}}{\sqrt{2}} \bar{\mu} \gamma_{\rho}\left(1-\gamma_{5}\right) \nu_{S} \bar{\nu}_{e} \gamma^{\rho}\left(1-\gamma_{5}\right) e \\
\mathcal{H}_{D}^{+} & =\frac{G_{F}}{\sqrt{2}} \cos \vartheta_{C} \bar{\nu}_{D} \gamma_{\lambda}\left(1-\gamma_{5}\right) e \bar{n} \gamma^{\lambda}\left(1-g_{A} \gamma_{5}\right) p
\end{aligned}
$$

describing muon and neutron decay, respecitively, and

$$
\nu_{S} \equiv U_{\mu j} \nu_{j}, \quad \nu_{D} \equiv U_{e j} \nu_{j}
$$

Actually, $\bar{\nu}_{e}$ in Eq.(2.4) should be replaced by $U_{e j}^{*} \bar{\nu}_{j}$, however, this has no effect on the final result for neutrinos much lighter than the mass of the muon. The Hamiltonians with the superscript + are the Hermitian conjugates of those which carry the minus sign.

Let us now sketch how to incorporate the finite muon lifetime in perturbation theory [20]. To this end we take into account $H_{D}$ only when it occurs together with $c_{i}(t)$ (see the initial conditions (2.7)) but take $H_{S}$ in all instances. With this proviso the following states and coefficients are involved in perturbation theory:

$$
\begin{array}{lrll}
\text { initial state } & \mu^{+} ; p & \leftrightarrow c_{i}, & \phi_{i} \\
\text { intermediate states } & e^{+}, \nu_{e}, \bar{\nu}_{S} ; p & \leftrightarrow c_{j}^{\prime}, \quad \phi_{j}^{\prime} \\
\mu^{+} ; e^{+}, n, \nu_{D} & \leftrightarrow c_{k}^{\prime \prime}, \quad \phi_{k}^{\prime \prime} \\
e^{+}, \nu_{e}, \bar{\nu}_{S} ; e^{+}, n, \nu_{D} & \leftrightarrow c_{j \otimes k}^{\prime \prime \prime}, \phi_{j \otimes k}^{\prime \prime \prime} \\
e^{+}, \nu_{e} ; e^{+}, n & \leftrightarrow c_{f}, \quad \phi_{f}
\end{array}
$$

The initial conditions for the coefficients are given by

$$
c_{i}(0)=1 \quad \text { and } \quad c_{f}(0)=c_{j}^{\prime}(0)=c_{k}^{\prime \prime}(0)=c_{j \otimes k}^{\prime \prime \prime}(0)=0 .
$$

The differential equations for the coefficients are

$$
\begin{aligned}
i \dot{c}_{i}(t) & \simeq \sum_{j} c_{j}^{\prime}(t)\left\langle\phi_{i} \mid H_{S, \text { int }}^{-}(t) \phi_{j}^{\prime}\right\rangle, \\
i \dot{c}_{f}(t) & =\sum_{j} c_{j}^{\prime}(t)\left\langle\phi_{f} \mid H_{D, \text { int }}^{+}(t) \phi_{j}^{\prime}\right\rangle+\sum_{k} c_{k}^{\prime \prime}(t)\left\langle\phi_{f} \mid H_{S, \text { int }}^{+}(t) \phi_{k}^{\prime \prime}\right\rangle, \\
i \dot{c}_{j}^{\prime}(t) & \simeq c_{i}(t)\left\langle\phi_{j}^{\prime} \mid H_{S, \text { int }}^{+}(t) \phi_{i}\right\rangle, \\
i \dot{c}_{k}^{\prime \prime}(t) & \simeq c_{i}(t)\left\langle\phi_{k}^{\prime \prime} \mid H_{D, \text { int }}^{+}(t) \phi_{i}\right\rangle+\sum_{j} c_{j \otimes k}^{\prime \prime \prime}(t)\left\langle\phi_{k}^{\prime \prime} \mid H_{S, \text { int }}^{-}(t) \phi_{j \otimes k}^{\prime \prime \prime}\right\rangle, \\
i \dot{c}_{j \otimes k}^{\prime \prime \prime}(t) & \simeq c_{k}^{\prime \prime}(t)\left\langle\phi_{j \otimes k}^{\prime \prime \prime} \mid H_{S, \text { int }}^{+}(t) \phi_{k}^{\prime \prime}\right\rangle .
\end{aligned}
$$


With these approximations we get a closed system for $c_{i}, c_{j}^{\prime}, c_{k}^{\prime \prime}, c_{j \otimes k}^{\prime \prime \prime}$. If we insert Eq.(2.12) into Eq.(2.11) we arrive at

$$
i \dot{c}_{k}^{\prime \prime}(t) \simeq c_{i}(t)\left\langle\phi_{k}^{\prime \prime} \mid H_{D, \text { int }}^{+}(t) \phi_{i}\right\rangle-i \sum_{j}\left\langle\phi_{k}^{\prime \prime} \mid H_{S, \text { int }}^{-}(t) \phi_{j \otimes k}^{\prime \prime \prime}\right\rangle \int_{0}^{t} d t^{\prime} c_{k}^{\prime \prime}\left(t^{\prime}\right)\left\langle\phi_{j \otimes k}^{\prime \prime \prime} \mid H_{S, \text { int }}^{+}\left(t^{\prime}\right) \phi_{k}^{\prime \prime}\right\rangle .
$$

However, looking at the intermediate states (2.6), we see that the equations

$$
\left\langle\phi_{j \otimes k}^{\prime \prime \prime} \mid H_{S, \text { int }}^{+}(0) \phi_{k}^{\prime \prime}\right\rangle=\left\langle\phi_{j}^{\prime} \mid H_{S, \text { int }}^{+}(0) \phi_{i}\right\rangle \quad \text { and } \quad E_{k}^{\prime \prime}-E_{j \otimes k}^{\prime \prime \prime}=E_{i}-E_{j}^{\prime}
$$

hold trivially because in the first matrix element $e^{+}, n, \nu_{D}$ and in the second second matrix element the proton are only spectators. Inserting Eq.(2.14) into Eq.(2.13) then with a partial integration the second term of Eq.(2.13) is written as

$$
-i \sum_{j} A_{j}\left[\left.\frac{e^{i\left(E_{i}-E_{j}^{\prime}\right)\left(t-t^{\prime}\right)}}{-i\left(E_{i}-E_{j}^{\prime}\right)} c_{k}^{\prime \prime}\left(t^{\prime}\right)\right|_{0} ^{t}-\int_{0}^{t} d t^{\prime} \frac{e^{i\left(E_{i}-E_{j}^{\prime}\right)\left(t-t^{\prime}\right)}}{-i\left(E_{i}-E_{j}^{\prime}\right)} \dot{c}_{k}^{\prime \prime}\left(t^{\prime}\right)\right]
$$

where

$$
A_{j} \equiv\left|\left\langle\phi_{j}^{\prime} \mid H_{S, \text { int }}^{+}(0) \phi_{i}\right\rangle\right|^{2}
$$

We neglect now the term with $\dot{c}_{j}^{\prime \prime}$ in Eq. 2.15) because it is of higher order and replace $E_{i}-E_{j}^{\prime}$ by $E_{i}-E_{j}^{\prime}+i \epsilon(\epsilon \downarrow 0)$ in Eq.(2.15) to have a well-defined expression. Since $c_{k}^{\prime \prime}(0)=0$ we obtain

$$
\begin{aligned}
& -\left.i \sum_{j} A_{j} \frac{e^{\left[i\left(E_{i}-E_{j}^{\prime}\right)-\epsilon\right]\left(t-t^{\prime}\right)}}{-i\left(E_{i}-E_{j}^{\prime}\right)+\epsilon} c_{k}^{\prime \prime}\left(t^{\prime}\right)\right|_{0} ^{t}=\sum_{j} A_{j} \frac{c_{k}^{\prime \prime}(t)}{E_{i}-E_{j}^{\prime}+i \epsilon} \\
= & \left(\sum_{j} A_{j} P\left(\frac{1}{E_{i}-E_{j}^{\prime}}\right)-i \pi \sum_{j} A_{j} \delta\left(E_{i}-E_{j}^{\prime}\right)\right) c_{k}^{\prime \prime}(t)=\left(\Delta E_{i}-\frac{i}{2} \Gamma\right) c_{k}^{\prime \prime}(t)
\end{aligned}
$$

where $P$ denotes the Cauchy's principal value and $\Gamma$ the total decay width of the muon. We neglect in the following the energy shift $\Delta E_{i}$ or we can think it being already incorporated in the muon mass. Hence we get

$$
i \dot{c}_{k}^{\prime \prime}(t) \simeq c_{i}(t)\left\langle\phi_{k}^{\prime \prime} \mid H_{D, \text { int }}^{+}(t) \phi_{i}\right\rangle-\frac{i}{2} \Gamma c_{k}^{\prime \prime}(t)
$$

With similar arguments one obtains [20]

$$
c_{i}(t) \simeq e^{-\frac{1}{2} \Gamma t}
$$

and therefore

$$
c_{k}^{\prime \prime}(t) \simeq-i \int_{0}^{t} d t^{\prime}\left\langle\phi_{k}^{\prime \prime} \mid H_{D, \text { int }}^{+}\left(t^{\prime}\right) \phi_{i}\right\rangle e^{-\frac{1}{2} \Gamma t} .
$$

Using Eqs. 2.9) and (2.10) we get the final result 


$$
\begin{aligned}
c_{f}(t) \simeq & (-i)^{2} \int_{0}^{t} d t_{1} \int_{0}^{t_{1}} d t_{2} \\
& \times\left\langle\phi_{f k} \mid\left(H_{D, \text { int }}^{+}\left(t_{1}\right) H_{S, \text { int }}^{+}\left(t_{2}\right) e^{-\frac{1}{2} \Gamma t_{2}}+H_{S, \text { int }}^{+}\left(t_{1}\right) e^{-\frac{1}{2} \Gamma t_{1}} H_{D, \text { int }}^{+}\left(t_{2}\right)\right) \phi_{i}\right\rangle .
\end{aligned}
$$

This formula corresponds to the intuitive expectation. Apart from starting the time integration at the inital time $t_{i}=0$ instead of $t_{i}=-\infty$ we have the usual time-ordered product with the finite lifetime incorporated in the exponentials.

\section{THE AMPLITUDE}

With Eq.2.21), the Hamiltonian densities (2.4) and Eq.(2.2) we can write for the amplitude of the process (1.5) in the limit $t \rightarrow \infty$

$$
\begin{aligned}
\mathcal{A}= & (-i)^{2}\left\langle\nu_{e}\left(p_{\nu}^{\prime}\right), e_{S}^{+}\left(p_{e S}^{\prime}\right) ; e_{D}^{+}\left(p_{e D}^{\prime}\right), n\left(p_{n}^{\prime}\right)\right| \\
& \times T\left[\int_{0}^{\infty} d t_{1} \int d^{3} x_{1} \int_{0}^{\infty} d t_{2} \int d^{3} x_{2} \mathcal{H}_{S, \text { int }}^{+}\left(x_{1}\right) e^{-\frac{1}{2} \Gamma t_{1}} \mathcal{H}_{D, \text { int }}^{+}\left(x_{2}\right)\right]\left|\mu^{+} ; p\right\rangle
\end{aligned}
$$

where $T$ is the time-ordering symbol. We assume that the muon $\mu^{+}$and the proton $p$ are localized at the coordinates $\vec{x}_{S}$ and $\vec{x}_{D}$, respectively. We imagine the proton being the nucleus of a hydrogen atom and bound in a molecule. Therefore we assume the proton state as stationary whereas the decaying muon will be described by a free wave packet with an average momentum equal to zero. This situation corresponds to the LSND experiment where the $\mu^{+}$is assumed to decay at rest. Since neutrino production and detection are localized at $\vec{x}_{S}$ and $\vec{x}_{D}$, respectively, the spinors of the initial particles can be written in coordinate space as

$$
\psi_{p}(x)=\psi_{p}\left(\vec{x}-\vec{x}_{D}\right) e^{-i E_{p} t}
$$

and

$$
\psi_{\mu}(x)=\int \frac{d^{3} p}{(2 \pi)^{3 / 2}} \widetilde{\psi}_{\mu}(\vec{p}) e^{-i\left(\vec{p} \cdot \vec{x}-E_{\mu}(\vec{p}) t\right)} \times e^{i \vec{p} \cdot \vec{x}_{S}},
$$

respectively, with $E_{\mu}(\vec{p})=\sqrt{m_{\mu}^{2}+\vec{p}^{2}}$. The function $\psi_{p}(\vec{y})$ is peaked at $\vec{y}=\overrightarrow{0}$ and the wave packet $\widetilde{\psi}_{\mu}(\vec{p})$ in momentum space is peaked around the average momentum $\langle\vec{p}\rangle=\overrightarrow{0}$. The final particles will be described by plane waves.

With the neutrino propagators of the mass eigenstate neutrinos

$$
\left\langle 0\left|T\left[\nu_{j}\left(x_{1}\right) \bar{\nu}_{j}\left(x_{2}\right)\right]\right| 0\right\rangle=i \int \frac{d^{4} q}{(2 \pi)^{4}} \frac{\not q+m_{j}}{q^{2}-m_{j}^{2}+i \epsilon} e^{-i q \cdot\left(x_{1}-x_{2}\right)}
$$

we obtain the amplitude 


$$
\begin{aligned}
\mathcal{A}= & (-i)^{2} \frac{G_{F}^{2} \cos \vartheta_{C}}{2} \int \frac{d^{3} p}{(2 \pi)^{3 / 2}} \int_{0}^{\infty} d t_{1} \int d^{3} x_{1} \int_{0}^{\infty} d t_{2} \int d^{3} x_{2} \int \frac{d^{4} q}{(2 \pi)^{4}} e^{-i q \cdot\left(x_{1}-x_{2}\right)} \\
& \times \exp \left\{i\left(p_{\nu}^{\prime}+p_{e S}^{\prime}\right) \cdot x_{1}+i\left(p_{n}^{\prime}+p_{e D}^{\prime}\right) \cdot x_{2}\right\} \exp \left\{i\left(\vec{p} \cdot \vec{x}_{1}-E_{\mu}(\vec{p}) t_{1}-\vec{p} \cdot \vec{x}_{S}\right)\right\} e^{-\frac{1}{2} \Gamma t_{1}} e^{-i E_{p} t_{2}} \\
& \times \bar{\psi}_{\mu}(\vec{p}) \gamma_{\rho}\left(1-\gamma_{5}\right) i \sum_{j} U_{\mu j} \frac{\not 1+m_{j}}{q^{2}-m_{j}^{2}+i \epsilon} U_{e j}^{*} \gamma^{\lambda}\left(1-\gamma_{5}\right) v_{e}\left(p_{e D}^{\prime}\right) \\
& \times J_{S}^{\rho}\left(p_{\nu}^{\prime}, p_{e S}^{\prime}\right) \bar{u}_{n}\left(p_{n}^{\prime}\right) \gamma_{\lambda}\left(1-g_{A} \gamma_{5}\right) \psi_{p}\left(\vec{x}_{2}-\vec{x}_{D}\right)
\end{aligned}
$$

with

$$
J_{S}^{\rho}\left(p_{\nu}^{\prime}, p_{e S}^{\prime}\right)=\bar{u}_{\nu_{e}}\left(p_{\nu}^{\prime}\right) \gamma^{\rho}\left(1-\gamma_{5}\right) v_{e}\left(p_{e S}^{\prime}\right)
$$

We start with the integration over $t_{1}$ where we have to calculate the integral

$$
\int_{0}^{\infty} e^{-i\left(q_{0}-E_{\nu}^{\prime}-E_{e S}^{\prime}+E_{\mu}(\vec{p})\right) t_{1}} e^{-\frac{1}{2} \Gamma t_{1}} d t_{1}=\frac{1}{i\left(q_{0}-E_{\nu}^{\prime}-E_{e S}^{\prime}+E_{\mu}(\vec{p})\right)+\frac{1}{2} \Gamma} .
$$

For the integration over $t_{2}$ we use the relation

$$
\lim _{t \rightarrow \infty} \int_{0}^{t} e^{i E t_{2}} d t_{2}=i P\left(\frac{1}{E}\right)+\pi \delta(E) .
$$

Hence a factor

$$
i P\left(\frac{1}{q_{0}+E_{n}^{\prime}+E_{e D}^{\prime}-E_{p}}\right)+\pi \delta\left(q_{0}+E_{n}^{\prime}+E_{e D}^{\prime}-E_{p}\right)
$$

appears in the amplitude. Furthermore, in the integration over $\vec{x}_{2}$ we use the relation

$$
(2 \pi)^{-3 / 2} \int d^{3} x e^{-i \vec{k} \cdot \vec{x}} f(\vec{x}+\vec{b})=e^{i \vec{k} \cdot \vec{b}} \tilde{f}(\vec{k})
$$

where $\tilde{f}$ is the Fourier transform of $f$. The integration over $\vec{x}_{1}$ is again trivial leading to the delta function

$$
(2 \pi)^{3} \delta\left(\vec{q}-\vec{p}_{\nu}^{\prime}-\vec{p}_{e S}^{\prime}+\vec{p}\right) .
$$

Thus we obtain

$$
\begin{aligned}
\mathcal{A}= & -\frac{G_{F}^{2} \cos \vartheta_{C}}{2} i \sum_{j} \int d^{3} p \int \frac{d^{4} q}{(2 \pi)^{4}} \exp \left\{-i \vec{p} \cdot \vec{x}_{S}-i\left(\vec{p}_{n}^{\prime}+\vec{p}_{e D}^{\prime}+\vec{q}\right) \cdot \vec{x}_{D}\right\} \\
& \times(2 \pi)^{3} \delta\left(\vec{q}-\vec{p}_{\nu}^{\prime}-\vec{p}_{e S}^{\prime}+\vec{p}\right) \frac{1}{i\left(q_{0}+E_{S}\right)+\frac{1}{2} \Gamma}\left[i P\left(\frac{1}{q_{0}+E_{D}}\right)+\pi \delta\left(q_{0}+E_{D}\right)\right] \\
& \times \bar{\psi}_{\mu}(\vec{p}) \gamma_{\rho}\left(1-\gamma_{5}\right) U_{\mu j} \frac{\not q+m_{j}}{q^{2}-m_{j}^{2}+i \epsilon} U_{e j}^{*} \gamma^{\lambda}\left(1-\gamma_{5}\right) v_{e}\left(p_{e D}^{\prime}\right) \\
& \times J_{S}^{\rho}\left(p_{\nu}^{\prime}, p_{e S}^{\prime}\right) \bar{u}_{n}\left(p_{n}^{\prime}\right) \gamma_{\lambda}\left(1-g_{A} \gamma_{5}\right) \widetilde{\psi}_{p}\left(\vec{q}+\vec{p}_{n}^{\prime}+\vec{p}_{e D}^{\prime}\right)
\end{aligned}
$$

where we have defined 


$$
E_{S} \equiv E_{\mu}(\vec{p})-E_{\nu}^{\prime}-E_{e S}^{\prime} \quad \text { and } \quad E_{D} \equiv E_{n}^{\prime}+E_{e D}^{\prime}-E_{p} .
$$

The integration over $\vec{p}$ can easily be carried out because of the delta function (3.11) and leads to the amplitude

$$
\begin{aligned}
\mathcal{A}= & -\frac{G_{F}^{2} \cos \vartheta_{C}}{2} e^{-i \vec{p}_{1} \cdot \vec{x}_{S}-i \vec{p}_{2} \cdot \vec{x}_{D}} i \sum_{j} \int \frac{d^{4} q}{2 \pi} e^{-i \vec{q} \cdot \vec{L}} \\
& \times \frac{1}{i\left(q_{0}+E_{S}\right)+\frac{1}{2} \Gamma}\left[\pi \delta\left(q_{0}+E_{D}\right)+i P\left(\frac{1}{q_{0}+E_{D}}\right)\right] \\
& \times \widetilde{\psi}_{\mu}\left(\vec{p}_{1}-\vec{q}\right) \gamma_{\rho}\left(1-\gamma_{5}\right) U_{\mu j} \frac{\not q+m_{j}}{q^{2}-m_{j}^{2}+i \epsilon} U_{e j}^{*} \gamma^{\lambda}\left(1-\gamma_{5}\right) v_{e}\left(p_{e D}^{\prime}\right) \\
& \times J_{S}^{\rho}\left(p_{\nu}^{\prime}, p_{e S}^{\prime}\right) \bar{u}\left(p_{n}^{\prime}\right) \gamma_{\lambda}\left(1-g_{A} \gamma_{5}\right) \widetilde{\psi}_{p}\left(\vec{q}+\vec{p}_{2}\right)
\end{aligned}
$$

where

$$
\vec{p}_{1} \equiv \vec{p}_{\nu}^{\prime}+\vec{p}_{e S}^{\prime}, \quad \vec{p}_{2} \equiv \vec{p}_{n}^{\prime}+\vec{p}_{e D}^{\prime} \quad \text { and } \quad \vec{L} \equiv \vec{x}_{D}-\vec{x}_{S}
$$

Note that as a consequence of the integration over $\vec{p}$ we have $E_{S}=E_{\mu}\left(-\vec{q}+\vec{p}_{1}\right)-E_{\nu}^{\prime}-E_{e S}^{\prime}$, i.e., $E_{S}$ is now a function of $\vec{q}$.

Now only the integration over $q$ remains. Since we have a delta function of $q_{0}$ within the brackets, the integration of the first of the two terms of the amplitude is trivial. We will show in the appendix that the $q_{0}$ integration in the second term, which contains Cauchy's principal value, leads in the limit of a macroscopic distance $L$ to the same result. In other words, in the limit of macroscopic $L$ we have simply $2 \pi \delta\left(q_{0}+E_{D}\right)$ from the $t_{2}$ integration. In this limit we can apply a theorem proved in Ref. 113 to perform the $d^{3} q$ integration and calculate the leading term of the amplitude for large $L$ :

$$
\begin{aligned}
\mathcal{A}^{\infty}= & \sum_{j} U_{\mu j} U_{e j}^{*} e^{i q_{j} L} \mathcal{A}_{j}^{\infty} \\
= & \frac{G_{F}^{2} \cos \vartheta_{C}}{2} \frac{2 \pi^{2}}{L} i \sum_{j} U_{\mu j} U_{e j}^{*} e^{i q_{j} L} \frac{1}{i\left(E_{S j}-E_{D}\right)+\frac{1}{2} \Gamma} \\
& \times \widetilde{\psi}_{\mu}\left(\vec{p}_{1}+q_{j} \vec{l}\right) \gamma_{\rho}\left(1-\gamma_{5}\right)\left(-k_{j}+m_{j}\right) \gamma_{\lambda}\left(1-\gamma_{5}\right) v_{e}\left(p_{e D}^{\prime}\right) \\
& \times J_{S}^{\rho}\left(p_{\nu}^{\prime}, p_{e S}^{\prime}\right) \bar{u}\left(p_{n}^{\prime}\right) \gamma^{\lambda}\left(1-g_{A} \gamma_{5}\right) \widetilde{\psi}_{p}\left(-q_{j} \vec{l}+\vec{p}_{2}\right)
\end{aligned}
$$

where the definition of $\mathcal{A}_{j}^{\infty}$ is obvious, $\vec{l}$ is the unit vector pointing from the neutrino source to the detection point, $k_{j}$ are the momenta of the intermediate neutrinos and

$$
k_{j} \equiv\left(\begin{array}{c}
E_{D} \\
q_{j} \vec{l}
\end{array}\right), \quad q_{j} \equiv \sqrt{E_{D}^{2}-m_{j}^{2}} .
$$

Note that

$$
E_{S j}=E_{\mu}\left(q_{j} \vec{l}+\vec{p}_{1}\right)-E_{\nu}^{\prime}-E_{e S}^{\prime}
$$

because by virtue of the theorem in Ref. [13] for each $j$ the vector $\vec{q}$ has to be replaced by $-q_{j} \vec{l}$. The irrelevant phase factor occurring in the first line of Eq. (3.14) has been dropped in $\mathcal{A}^{\infty}$. 


\section{COHERENCE CONDITIONS INDEPENDENT OF $L$}

Inspecting Eq.(3.16) it is evident that oscillations involving $m_{j}^{2}-m_{k}^{2}$ can only take place if 3,13

$$
\left|q_{j}-q_{k}\right| \lesssim \sigma_{S} \quad \text { and } \quad\left|q_{j}-q_{k}\right| \lesssim \sigma_{D}
$$

where $\sigma_{S}$ and $\sigma_{D}$ are the widths of $\widetilde{\psi}_{\mu}$ and $\widetilde{\psi}_{p}$, respectively. We call conditions (4.1) amplitude coherence conditions (ACC). If they are not fulfilled, either by the source wave packet or the detector wave packet, then $\mathcal{A}_{j}^{\infty} \times \mathcal{A}_{k}^{\infty} \approx 0(j \neq k)$ which means that the term labelled by $j k$ is suppressed in Eq.(1.1). In the ultrarelativistic limit Eq.(4.1) is rephrased as

$$
\frac{\Delta m_{j k}^{2}}{2 E_{D}} \lesssim \sigma_{S, D}
$$

Denoting by $\sigma_{x S, x D}$ the widths of the wave functions $\psi_{\mu}(x)$ and $\psi_{p}(x)$ in coordinate space, respectively, then, with the uncertainty relations $\sigma_{x S, x D} \sigma_{S, D} \geq 1 / 2$, Eq.(4.2) is rewritten as

$$
\sigma_{x S, x D} \lesssim \frac{1}{4 \pi} L_{j k}^{\mathrm{osc}}
$$

where we have made the identification $E_{\nu}=E_{D}$ (see Eqs.(3.16) and (3.17)).

The amplitude $\mathcal{A}_{j}^{\infty}$ contains the factor

$$
\frac{1}{i\left(E_{S j}-E_{D}\right)+\frac{1}{2} \Gamma}
$$

which leads to a condition analogous to Eq.(4.1) for neutrino oscillations to take place:

$$
\left|E_{S j}-E_{S k}\right| \lesssim \frac{1}{2} \Gamma
$$

In the following this condition will be called source wave packet - finite lifetime condition (SFC). In the ultrarelativistic limit and with $\sigma_{S} \ll m_{\mu}$ we obtain

$$
E_{S j}-E_{S k} \approx-\frac{\Delta m_{j k}^{2}}{2 m_{\mu} E_{D}}\left(E_{D}+\vec{l} \cdot \vec{p}_{1}\right)
$$

and, assuming that ACC holds, we observe that $\left|E_{D} \vec{l}+\vec{p}_{1}\right| \lesssim \sigma_{S}$ is valid (see the argument of $\widetilde{\psi}_{\mu}$ in $\mathcal{A}^{\infty}(3.16)$ ) and therefore Eq.(4.5) is rewritten as

$$
\frac{\Delta m_{j k}^{2} \sigma_{S}}{m_{\mu} E_{D}} \lesssim \Gamma \text {. }
$$

Defining $\Delta v_{\mu} \equiv \sigma_{S} / m_{\mu}$ as a measure for the spread in velocity of the muon wave packet and with the muon lifetime $\tau_{\mu}=1 / \Gamma$ we can interpret Eq.(4.7) as

$$
\Delta v_{\mu} \tau_{\mu} \lesssim \frac{1}{4 \pi} L_{j k}^{\mathrm{osc}} .
$$




\section{THE COHERENCE LENGTH DUE TO THE FINITE $\mu$ LIFETIME}

Having performed all the integrations in the amplitude in the limit $L \rightarrow \infty$, we will discuss some aspect of the integration in the cross section. There we have integrations of the form $d^{3} p^{\prime} / 2 E^{\prime}$ for each particle in the final state, i.e., in our case $\nu_{e}$ and $e_{S}^{+}$in the source process and $n$ and $e_{D}^{+}$in the detector process. In general these integrations cannot be performed without knowledge of the source and detector wave functions. However, for

$$
\Gamma \ll \sigma_{S, D}
$$

the factors

$$
\left\{\left(i\left(E_{S j}-E_{D}\right)+\Gamma / 2\right) \times\left(-i\left(E_{S k}-E_{D}\right)+\Gamma / 2\right)\right\}^{-1}
$$

in the cross section can be used to apply Cauchy's Theorem in order to obtain the coherence length associated with the finite muon lifetime. We assume that the ACC and SFC are valid and integrating over momenta of the final state of the detector leads to an integration in the variable $E_{D}$ over a particular interval containing $E_{S j} \forall j$ such that the length $\Delta E_{D}$ of this interval fulfills $\Gamma \ll \Delta E_{D} \ll \sigma_{S, D}$. This allows to write $E_{D}=\bar{E}_{D}+\varepsilon$ where $\bar{E}_{D}$ denotes the central value of the interval which we define as

$$
\bar{E}_{D} \equiv\left\langle E_{S j}\right\rangle
$$

where $\left\langle E_{S j}\right\rangle$ denotes the mean value of the $E_{S j}$ and the integration variable $\varepsilon$ varies over $|\varepsilon| \leq \Delta E_{D} / 2$. From the SFC it follows that $\bar{E}_{D} \approx E_{S j} \forall j$ which is exact up to terms of order $\Gamma$. With $\Delta m_{j k}^{2}>0$ and $q_{j}-q_{k} \approx-\Delta m_{j k}^{2} / 2 E_{D}$ we observe that the $\varepsilon$ integration over the interval on the real axis can be closed via a half-circle in the upper half-plane since the radius of the half-circle is much larger than $\Gamma$ and therefore the factors (5.2) make the part of the path in the upper half-plane negligible in the integral. Because of Eq.(5.1) and $\Delta E_{D} \ll \sigma_{S, D}$ this integration does not affect the source and detector wave functions to a good approximation. Then Cauchy's Theorem states that the result of the integration is given by making the replacement

$$
E_{D} \rightarrow E_{D}^{(0)}=\bar{E}_{D}+\frac{i}{2} \Gamma
$$

in the absolute square of $\mathcal{A}^{\infty}(3.16)$. Inserting $E_{D}^{(0)}$ into the exponential $-i \Delta m_{j k}^{2} L / 2 E_{D}$ we see that the cross section contains the damping factor

$$
\exp \left(-\frac{\Delta m_{j k}^{2} \Gamma}{4 \bar{E}_{D}^{2}} L\right)
$$

As we will discuss in the next section the requirement (5.1) is very likely to be fulfilled for LSND with the decay width of the muon being $\Gamma \approx 3 \times 10^{-16} \mathrm{MeV} . \bar{E}_{D}$ can still be thought of as being identical with $E_{D}$ (3.13) for all practical purposes because this $\Gamma$ is so small that $\Delta E_{D}$ can be chosen smaller than any achievable accuracy for $E_{D}$ in a real experiment. 


\section{DISCUSSION}

The characteristics of the field-theoretical approach: In this paper we have used the field-theoretical approach to discuss neutrino oscillations as we have done in Ref. [13]. In this approach the whole process of neutrino production and detection is represented by a single Feynman graph such that the oscillating neutrinos are associated with the inner line of the graph. However, due to the macroscopic distance between neutrino source and detector this inner line is on-shell (see Eq.(3.16)) for each neutrino with definite mass according to a theorem proven in Ref. [13]. In the present paper we have incorporated the finite lifetime of the neutrino source which is given in our concrete example of the LSND experiment (see Eq.(1.5) ) by a positively charged muon whose decay is responsible for the $\bar{\nu}_{\mu}$ neutrinos with which the experiment is performed. The finite lifetime of the neutrino source prevented us from using ordinary perturbation theory with an initial time $t_{i}=-\infty$. Instead we took advantage of the Weisskopf-Wigner approximation which allowed us to take $t_{i}=0$ and to combine in this way the finite muon lifetime with perturbation theory.

With the help of the above-mentioned theorem all integrations in the amplitude of the combined production - detection process could be performed in the asymptotic limit $L \rightarrow \infty$. From the requirement $\mathcal{A}_{j}^{\infty} \approx \mathcal{A}_{k}^{\infty}(3.16)$ we derived the amplitude coherence conditions (ACC) (4.1), (4.2) and the source wave packet - finite liftime condition (SFC) (4.5), (4.7). For a given mass-squared difference $\Delta m_{j k}^{2}$ these three conditions are the prerequisites for neutrino oscillations. If they are not fulfilled, the term with $\exp \left(-i \Delta m_{j k}^{2} L / 2 E_{\nu}\right)$ is suppressed in the oscillation probability which means that no neutrino oscillations with respect to $\Delta m_{j k}^{2}$ are possible. Here we have identified the neutrino energy $E_{\nu}$ with $E_{D}(3.13)$ which is justified in view of the definition of $q_{j}$ (3.17) occurring in the exponentials $e^{i q_{j} L}$ of $\mathcal{A}^{\infty}$ (3.16). ACC and SFC are both independent of $L$, therefore no coherence lengths are associated with them. In coordinate space Eq.(4.1) simply means that the oscillation length must be larger than the widths of the production and detection wave functions (see Eq.(4.3)). The ACC were among the main results of Refs. [3] and [13] and an analogous condition in the framework of the wave packet approach was recently emphasized in Ref. [12]. The SFC (4.5) says that the spreading of the muon wave function ${ }^{\text {[ }}$ during the muon lifetime should be less than the oscillation length in order not to wash out neutrino oscillations (see Eq.(4.8)). In Ref. [13] this condition was not found because it was assumed that the source wave function is stationary. Clearly, in such a case the energy of the neutrino source is fixed and does not depend on $m_{j}$ and, consequently, no SFC is present.

Discussion of the LSND experiment: Coming back to the LSND experiment, $\sigma_{S}$ represents the momentum spread of the stopped muon and an estimate of it is given by $\sigma_{S} \lesssim 0.01 \mathrm{MeV}$ [21]. For the detector proton bound in $\mathrm{CH}_{2}$ groups (mineral oil) [18 it is reasonable to assume that in coordinate space its wave function has a spread of around $1 \AA$ and consequently $\sigma_{D} \sim 2 \times 10^{-3} \mathrm{MeV}$. Dropping now the indices of the masssquared difference, with representative values $\Delta m^{2} \sim 1 \mathrm{eV}^{2}$ and $E_{\nu} \sim 30 \mathrm{MeV}$ we obtain $\Delta m^{2} / 2 E_{\nu} \sim 10^{-14} \mathrm{MeV}$ and we conclude that the amplitude coherence conditions are very

\footnotetext{
${ }^{3}$ The muon wave function is non-stationary.
} 
well fulfilled in the LSND experiment. Performing an analogous estimate with the SFC we get $\Delta m^{2} \sigma_{S} / m_{\mu} E_{\nu} \lesssim 3 \times 10^{-18} \mathrm{MeV} \ll \Gamma \approx 3 \times 10^{-16} \mathrm{MeV}$ with the numbers we used before for the ACC. From this result we conclude that also the SFC is valid in the context of the LSND experiment, though, surprisingly, the margin is only given by two orders of magnitude. Note that for the ACC this margin is eleven orders of magnitude.

In Ref. [19] it was found that $\Delta m^{2} / E_{\nu} \lesssim \Gamma$ should hold for coherent neutrino oscillations. This condition is not fulfilled for LSND. In this paper with the field-theoretical treatment we could not recover this condition which looks like the first ACC (4.2) with $\sigma_{S}$ replaced by $\Gamma$. In this context we want to stress the following: in the process (1.5) there are three different lengths, namely the sizes of the wave packets of the neutrino source of order $1 / \sigma_{S}$ and of the detector particle of order $1 / \sigma_{D}$, where $\sigma_{S}$ and $\sigma_{D}$ are the widths of $\widetilde{\psi}_{\mu}$ and $\widetilde{\psi}_{p}$, respectively, and the length $1 / \Gamma$ associated with the finite lifetime of the source. Each of these lengths is uniquely defined and the function of each of them uniquely determined in the field-theoretical framework. However, in the wave packet approach the distinction between $\sigma_{S}, \sigma_{D}$ and $\Gamma$ is not so clear and each of the three lengths could be associated with the size of the neutrino wave packet and possibly lead to erroneous conclusions.

The characteristics of neutrino oscillations: We want to emphasize that in the fieldtheoretical approach the notion of a neutrino wave packet does not exist and the questions whether the neutrino energy or neutrino momentum is fixed or both can vary do only make sense in connection with the processes of production and detection. This is because only parameters associated with particles of the exterior legs of the Feyman graph, i.e, with those particles which are manipulated in the experiment, determine the neutrino oscillations. Let us notice that, for fixed momenta of the final state particles of the production and the detection processes, with Eq.(3.16) the oscillation probability has the form

$$
P_{\bar{\nu}_{\mu} \rightarrow \bar{\nu}_{e}}(L) \propto\left|\sum_{j} \mathcal{A}_{j}^{\infty} U_{\mu j} U_{e j}^{*} e^{i q_{j} L}\right|^{2}
$$

and we can imagine that the phase factors $e^{i q_{j} L}$ represent the plane waves of the different neutrinos mass eigenstates. We will use this fact to compare the wave packet approach with the result of this paper. We arrive at the following characteristics of neutrino oscillations in our field-theoretical approach:

1. We have chosen the detector wave function, i.e., the wave function of the proton, to be a bound state and therefore the detector wave function does not spread in time. This looks physically very reasonable to us meaning that the detector is always on and waiting (see, however, Ref. [15] for a discussion of source and detector with a temporal resolution). As a consequence we have $E_{\nu}=E_{D}$ (see Eqs.(3.13), (3.16) and (3.17)) and Eq.(6.1) suggests that neutrino oscillations take place between neutrinos with the same energy but with different momenta $q_{j}$ 13.

2. The identification $E_{\nu}=E_{D}$ allows to determine the neutrino energy with arbitrary accuracy by measuring the energies of the particles in the final state of the detector process, in our case the neutron and the detector positron, with arbitrary accuracy. Therefore, one can limit the averaging over the neutrino energy to an arbitrarily small 
interval - of course, in practice at the expense of the number of events - and make any coherence length arbitrarily long by performing only detector manipulations (see Eq.(1.3)). This so-called restauration of coherence is trivial in our approach. It agrees with observations in the wave packet treatment [0, 11]. We find no upper limit to the coherence lengths in contrast to Ref. [12] which is due to the fact that we assume that the detector is sensitive to energies and momenta of the particles produced in the detector process whereas in Ref. [12] it is assumed that the detector measures neutrino wave packets of a certain width.

3. Taking the field-theoretical approach to neutrino oscillations seriously, assuming that the detector particle is initially in a stationary state and that the observation of particles associated with the neutrino detection is done by energy and momentum measurements, we come to inevitable conclusion that there are no neutrino wave packets in neutrino oscillations because all summations over neutrino energies happen in the cross section and are thus incoherent summations.f Our assumptions include the cases that some particles are not detected at all or that cuts in energy and momentum are made. In addition, any further measurements of observables commuting with the energy and momentum operators performed by the detector do not change our conclusion [0]. We think that our conclusion is correct for realistic experiments. If one assumes instead that, with respect to the particles in the final state of the detection process, the detector is sensitive to wave packets of some form then our conclusion is not valid. However, we do not know if such a detector exists.

The coherence lengths: Let us now assume that the ACC and SFC hold and study the effects of different energy and/or momentum averaging mechanisms which all lead to specific coherence lengths. In the light of the above discussion all coherence lengths result from incoherent neutrino energy spreads. There are three types of coherence lengths [7] associated with our neutrino production and detection processes (1.5):

A. $L_{A}^{\text {coh }}$ due to the finite muon lifetime,

B. $L_{B}^{\text {coh }}$ due to the interruption of the neutrino emission because of collisions of the source positron with the background and

C. $L_{C}^{\text {coh }}$ due to the neutrino energy spread introduced by the usual imperfect energy measurements of the particles observed with a realistic detector.

According to Eq. (5.5) the first of the coherence lengths is given by

$$
L_{A}^{\mathrm{coh}}=\frac{4 E_{\nu}^{2}}{\Delta m^{2} \Gamma} .
$$

\footnotetext{
${ }^{4}$ Though in this paper we consider a neutrino source at rest, this conclusion and points 1,2 are also valid for accelerator neutrinos because the arguments leading to it depend on the neutrino detection process and not on the production. Formulated in another way, it is the detector which through its properties determines the nature of the neutrino energy spread.
} 
This coherence length appears if the energy averaging amounts to a summation of $E_{D}$ over an interval much larger than $\Gamma$ (see section $\mathrm{V}$ ). With $1 / \Gamma$ corresponding to $659 \mathrm{~m}$ and $\Delta m^{2} \sim 1 \mathrm{eV}^{2}, E_{\nu} \sim 30 \mathrm{MeV}$ this coherence length is around 200 light years and completely irrelevant for the LSND experiment and, therefore, apart from its effect in the SFC, the finite lifetime of the muon could have been neglected as was done in Ref. [13] with the lifetime of the neutrino source nucleus. However, the main point in the investigation of $L_{A}^{\text {coh }}$ was rather to see how it emerges in the field-theoretical treatment. It is interesting to note that $L_{A}^{\text {coh }}$ enters into the cross section through $\exp \left(-L / L_{A}^{\mathrm{coh}}\right)$. We have obtained this form of the damping factor for $\Gamma \ll \sigma_{S}, \sigma_{D_{\tilde{\sim}}}$. If this condition is not fulfilled the damping factor depends on the form of the functions $\widetilde{\psi}_{\mu}, \widetilde{\psi}_{p}$. In the approach using Gaussian wave packets a corresponding damping factor has the form $\exp \left\{-\left(L / L^{\text {coh }}\right)^{2}\right\}$ [12], however, it is not obvious how to compare this factor with the previous one and which coherence length is meant with $L^{\mathrm{coh}}$.

$L_{B}^{\text {coh }}$ is not included in our treatment because we do not know how to deal with random collisions of the source positron with the matter background and the associated heuristic coherence length (1.3) in the field-theoretical approach. So here we only repeat the arguments found in the literature about the neutrino wave packet approach. There one estimates the mean free path of the positron from the $\mu^{+}$decay in the matter background where $\mu^{+}$is produced. In the LSND experiment this background consists of water 18 and according to the rule of thumb presented in Ref. [2] this mean free path of the positron is of the order of centimeters. Then in the heuristic approach one would estimate the size $\ell_{B}$ of the neutrino wave packet to be of the same order of magnitude [2]. Thus, adopting the wave packet approach, one would estimate $L_{B}^{\text {coh }} \sim E_{\nu}^{2} \ell_{B} / \Delta m^{2}$ which is something like four orders of magnitude smaller than $L_{A}^{\text {coh }}$, but still astronomical, making the previous consideration of the coherence length originating from the finite muon lifetime (or a wave packet size of order $\ell_{A} \sim c \tau_{\mu}=659 \mathrm{~m}$ ) spurious.

The coherence length $[7] L_{C}^{\text {coh }}=L^{\text {osc }} E_{\nu} / \Delta E_{\nu}$, where $\Delta E_{\nu}$ comes from the inability to measure $E_{\nu}=E_{D}$ better than at a certain realistic experimental accuracy, is the only relevant coherence length in practice [17]. In the LSND experiment [18] the detector positron and neutron are detected in coincidence and, in addition, the energy of the positron is measured. We guess that $\Delta E_{\nu}$ is of the order of $5 \mathrm{MeV}$ [18, thus $L_{C}^{\text {coh }}$ is probably not more than several times the oscillation length. This is many orders of magnitude smaller than the astronomical coherence lengths $L_{A, B}^{\text {coh }}$.

Summary: Let us summarize the main points. We have confined ourselves to situations where the neutrino source is at rest, thus the present investigation is not straightforwardly applicable to high energy neutrinos (see, however, Ref. [14] for a field-theoretical discussion of accelerator neutrino experiments). We have assumed that the wave function of the detector particle is stationary. Then the field-theoretical approach to neutrino oscillations is completely static and there are no explicit time averages necessary as in the wave packet

\footnotetext{
${ }^{5}$ Note that even this coherence length could theoretically be overcome by measuring $E_{D}$ with a precision better than $\Gamma$ and chosing events with $E_{D}$ in a given interval of size $\Delta E_{D}$ smaller than $\Gamma$ (compare section $\square$ and point 2 in this section).
} 
approach. With the field-theoretical method we have clarified, using the model reaction (1.5), the roles of the widths $\sigma_{S}, \sigma_{D}$ and $\Gamma$ in neutrino oscillations where these widths are associated with the source, the detector and the finite lifetime of the source, respectively. To check the validity of the ACC (4.2) and the SFC (4.7) in a real experiment concrete values of $\sigma_{S}$ and $\sigma_{D}$ have to be chosen. Making a plausible guess for $\sigma_{D}$ and using $\sigma_{S} \lesssim 0.01$ $\mathrm{MeV}$ [21] in the case of LSND, the ACC are very well fulfilled and also the SFC seems to hold safely. Finally, if our method is a correct approach to neutrino oscillations then, in experiments with the realistic detector properties assumed in this paper, oscillations take place between neutrino mass eigenstates with the same energy but different momenta, there are no neutrino wave packets and the coherence length in neutrino oscillations results from an incoherent neutrino energy spread.

\section{ACKNOWLEDGMENTS}

W.G. and S.M. thank the organizers of the 5th Workshop on High Energy Physics Phenomenology, January 12-26, 1998, Pune, India and the Theory Division of PRL - Ahmedabad for providing the opportunity to start this collaboration. S.M. thanks also S. Pakvasa and L.M. Sehgal for useful discussions. Furthermore, we are indebted to T. Goldman and W.C. Louis for information on the momentum spread of the stopped muons in the LSND experiment. 


\section{APPENDIX A: INTEGRATION OVER $q_{0}$}

We consider the integrals

$$
I_{n}=i \int_{-\infty}^{\infty} d q_{0} \frac{1}{i\left(q_{0}+E_{S}\right)+\frac{1}{2} \Gamma} P\left(\frac{1}{q_{0}+E_{D}}\right) \frac{\left(q_{0}\right)^{n}}{q_{0}^{2}-\vec{q}^{2}-m_{j}^{2}+i \epsilon}
$$

where $n=0,1$ and $P$ denotes Cauchy's principal value. We will calculate this integral with the help of the residue calculus which gives the formula

$$
\int d x P\left(\frac{1}{x-x_{0}}\right) f(x)=\frac{1}{2}\left(\int_{C_{R}}+\int_{C_{L}}\right) d x \frac{1}{x-x_{0}} f(x)
$$

for a function $f$ which is analytic along the real axis. The paths $C_{R}$ and $C_{L}$ lead along the real axis except close to $x=x_{0}$ where the point $x=x_{0}$ is circumvented to its right or to its left in the complex plane, respectively. In our case $x_{0}=-E_{D}$ and $f$ has three poles of first order at

$$
q_{0}^{(1)}=-E_{S}+\frac{i}{2} \Gamma, \quad q_{0}^{(2)}=\sqrt{\vec{q}^{2}+m_{j}^{2}}-i \epsilon \quad \text { and } \quad q_{0}^{(3)}=-\sqrt{\vec{q}^{2}+m_{j}^{2}}+i \epsilon .
$$

Since we have only one pole below the real axis we close the contour below. Then we obtain

$$
I_{n}=-\pi i\left\{\frac{x_{0}^{n}}{\left(x_{0}-q_{0}^{(1)}\right)\left(x_{0}-q_{0}^{(2)}\right)\left(x_{0}-q_{0}^{(3)}\right)}+\frac{2\left(q_{0}^{(2)}\right)^{n}}{\left(q_{0}^{(2)}-x_{0}\right)\left(q_{0}^{(2)}-q_{0}^{(1)}\right)\left(q_{0}^{(2)}-q_{0}^{(3)}\right)}\right\}
$$

In the second part of Eq.(A4) we use that $E_{D}>0$ and perform the limit $\epsilon \rightarrow 0$ without getting a singular integrand. The integral $I_{n}$ depends on $\vec{q}$ and appears in the amplitude in the following way:

$$
\mathcal{A}=\int d^{3} q \Phi(\vec{q}) I(\vec{q}) e^{-i \vec{q} \cdot \vec{L}}
$$

where $\Phi$ can be read off from Eq.(3.14). For the second term of $I_{n}$ we can use Lemma 3 of Ref. 113 to show that it decreases like $L^{-2}$ for $L \rightarrow \infty$. Since we are only interested in the leading term $\propto L^{-1}$ of the amplitude for large $L$ we can neglect the contribution of the second term of $I_{n}$. It is then easy to show that the first term in the integral (A4) gives exactly the contribution to the amplitude $\mathcal{A}^{\infty}$ (3.16) as the term with $\pi \delta\left(q_{0}+E_{D}\right)$. 


\section{REFERENCES}

[1] B. Pontecorvo, Sov. Phys. JETP 26, 984 (1968); S.M. Bilenky and B. Pontecorvo, Phys. Rep. 41, 225 (1978); S.M. Bilenky and S.T. Petcov, Rev. Mod. Phys. 59, 671 (1987); B. Kayser, F. Gibrat-Debu and F. Perrier, "The Physics of Massive Neutrinos", World Scientific, Singapore, 1989; R.N. Mohapatra and P.B. Pal, "Massive Neutrinos in Physics and Astrophysics", World Scientific Lecture Notes in Physics, Vol. 41, World Scientific, Singapore, 1991.

[2] C.W. Kim and A. Pevsner, "Neutrinos in Physics and Astrophysics", Contemporary Concepts in Physics, Vol. 8, Harwood Academic Press, Chur, Switzerland, 1993.

[3] J. Rich, Phys. Rev. D 48, 4318 (1993).

[4] S. Nussinov, Phys. Lett. 28B, 201 (1976).

[5] B. Kayser, Phys. Rev. D 24, 110 (1981).

[6] C. Giunti, C.W. Kim and U.W. Lee, Phys. Rev. D 44, 3635 (1991).

[7] K. Kiers, S. Nussinov and N. Weiss, Phys. Rev. D 53, 537 (1996).

[8] J. Lowe, B. Bassalleck, H. Burkhardt, A. Rusek, G.J. Stephenson Jr. and T. Goldman, Phys. Lett. B 384, 288 (1996); H. Burkhardt, J. Lowe, G.J. Stephenson Jr. and T. Goldman, hep-ph/9803365.

[9] B. Ancochea, A. Bramon, R. Muñoz-Tapia and M. Nowakowski, Phys. Lett. B 389, 149 (1996).

[10] Y. Grossman and H. Lipkin, Phys. Rev. D 55 (1997) 2760.

[11] C. Giunti, C.W. Kim and U.W. Lee, Phys. Lett. B 421, 237 (1998).

[12] C. Giunti and C.W. Kim, Phys. Rev. D 58, 017301 (1998).

[13] W. Grimus and P. Stockinger, Phys. Rev. D 54, 3414 (1996).

[14] J.E. Campagne, Phys. Lett. B 400, 135 (1997).

[15] K. Kiers and N. Weiss, Phys. Rev. D 57, 3091 (1998).

[16] C. Giunti, C.W. Kim, J.A. Lee and U.W. Lee, Phys. Rev. D 48, 4310 (1993).

[17] M. Fukugita, in "Physics and Astrophysics of Neutrinos", edited by M. Fukugita and A. Suzuki, Springer, Tokyo, Japan, 1994.

[18] LSND Coll., C. Athanassopoulos et. al., Phys. Rev. Lett. 77, 3082 (1996); Phys. Rev. C 54, 2685 (1996).

[19] S. Mohanty, preprint hep-ph/9710284.

[20] V. Weisskopf and E. Wigner, Z. Phys. 63, 54 (1930); ibid. 65, 18 (1930).

[21] W.C. Louis (LSND Coll.), private communication. 